\title{
REGULAR BISIMPLE RINGS
}

\author{
by JOHN HANNAH
}

(Received 15th March 1989)

\begin{abstract}
We characterize regular bisimple rings in terms of some perspectivity conditions on their lattices of principal right ideals. We also show that, if $S$ is the multiplicative subsemigroup generated by all the idempotents of a regular bisimple ring $R$, then

(i) if $R$ does not have an identity, then $S=R$ and has depth 2;

(ii) if $R$ does have an identity but is not a division ring, then $S=\{a \in R: a$ is neither left nor right invertible $\} \cup\{1\}$ and has depth 3 .
\end{abstract}

1980 Mathematics subject classification (1985 Revision): 16A30, $20 \mathrm{M} 17$.

\section{Introduction}

A ring $R$ is said to be bisimple if it has more than one element, and satisfies the following conditions:

(B1) for any $a \in R$ we have $a \in a R \cap R a$, and

(B2) for any nonzero $a, b \in R$ there is some $c \in R$ such that $a R=c R$ and $R c=R b$.

These rings were introduced by Munn [9] in 1981, and are precisely those rings whose multiplicative semigroups are 0-bisimple [9, Appendix]. Bisimple rings are always simple and are (von Neumann) regular as long as they contain a nonzero idempotent [9, Lemmas 1.2 and 1.3].

Let $R$ be a regular ring and $L(R)$ its lattice of principal right ideals. Thus $L(R)$ is a relatively complemented modular lattice. Munn showed that if $R$ is bisimple, then any two intervals in $L(R)$ are lattice-isomorphic [9, Theorem 2.1], and he went on to ask whether every relatively complemented, modular lattice $L$ with this property can be viewed as $L(R)$ for some regular bisimple ring $R$. In Section 1 of this paper we give an example (Example 1.1) of such a lattice $L$ which is not isomorphic to $L(R)$ for any regular bisimple ring $R$, thus answering Munn's question in the negative. We then find two lattice-theoretic characterizations of regular bisimple rings (Theorem 1.3), and use these to identify the complemented modular lattices which can arise as $L(R)$ for some regular bisimple ring $R$ with identity (Corollary 1.6).

Munn's paper essentially discusses the ring-theoretic consequences of imposing the semigroup condition, 0-bisimplicity, on the multiplicative semigroup of a ring. In Section 2 we take another point of view and consider some semigroup-theoretic consequences. Specifically, we study the multiplicative subsemigroup $S$ generated by all 
the idempotents of a regular bisimple ring $R$. We show, in Proposition 2.2, that if $R$ does not have an identity element, then every element of $R$ is a product of two idempotents (so that $S=R$ and has depth 2). On the other hand, if $R$ does have an identity but is not a division ring, then (Proposition 2.3) every element of $R$ which is neither left nor right invertible is a product of three idempotents (so that $S$ has depth exactly 3). These results may be compared with the situation where $R$ is an arbitrary regular 0-bisimple semigroup: in that setting it is possible for $S$ to have arbitrarily large depth.

\section{Preliminaries}

All rings in this paper are associative, but they need not have an identity element. If $a$ is an element of a ring $R$, then we write $r(a)$ for its right annihilator $\{s \in R: a s=0\}$. Similarly $l(a)$ denotes the left annihilator of $a$ in $R$.

A ring $R$ is (von Neumann) regular if for any $a \in R$ there is some $x \in R$ with $a=a x a$. Notice that if $a=a x a$ in a ring $R$ then $e=a x$ and $f=x a$ are idempotents such that $a R=e R$ and $R a=R f$. Furthermore, $l(a)=R(1-e)$ and $r(a)=(1-f) R$, (if $R$ does not have an identity, we interpret $R(1-e)$ as $\{s-s e: s \in R\}$, and similarly for $(1-f) R)$. Thus if $a, b$ are elements of a regular ring $R$, we have $R a=R b$ if and only if $r(a)=r(b)$. In particular, if $R$ is a regular ring with an identity element and $a \in R$, then $a$ is left invertible if and only if $r(a)=0$. Similarly, $a$ is right invertible if and only if $l(a)=0$.

A regular ring $R$ is abelian if all its idempotents are central in $R$. For basic results about regular rings and for any unexplained notation we refer the reader to Goodearl's book [1].

We say that a modular lattice $L$ is relatively complemented if, for any $A, B \in L$ with $A \leqq B$, there is some $C \in L$ with $A+C=B$ and $A \cap C=0$ (that is, $C$ is a complement of $A$ in $B$ ). If $L$ has a greatest element, then $L$ is relatively complemented if and only if it is complemented. If $L$ is a relatively complemented modular lattice, we say that $A, B \in L$ are perspective in $L$ if $A$ and $B$ have a common complement in $A+B$; that is, if there is some $C \in L$ such that

$$
A+C=B+C=A+B
$$

and

$$
A \cap C=0=B \cap C .
$$

If $L$ has a greatest element (and so is complemented), this definition agrees with that in [11] because of [11, Theorem 3.1, p. 17]. If $L$ is in fact the lattice $L R$ ) of principal right ideals of a regular ring $R$, then any pair of perspective elements $A, B$ in $L(R)$ are isomorphic as right $R$-modules, since if $C$ is as above, then

$$
A \cong \frac{A+C}{C}=\frac{B+C}{C} \cong B
$$


For other basic facts about lattices we refer the reader to [11]. Notice however that, because we shall always have in mind the lattices $L(R)$, we shall use + and $\cap$ to indicate the lattice operations. Furthermore, if $A$ and $B$ are independent elements of a lattice (that is, if $A \cap B=0$ ), we shall write their supremum in the lattice as $A \oplus B$ rather than simply $A+B$, (this agrees with the usage for $L(R)$ in [1]).

Finally for any unexplained terminology or basic results about semigroups, see Howie's book [5].

\section{Lattice characterizations of regular bisimple rings}

We begin this section with the example mentioned in the introduction.

Example 1.1. There is a complemented modular lattice $L$ in which any two intervals are lattice-isomorphic, but for which there is no regular bisimple ring $R$ such that $L \cong L(R)$.

Proof. We construct $L$ as the lattice of principal right ideals of a suitable regular ring $T$. Choose any field $F$, and for each positive integer $n$, set $F_{n}=F$. Consider the direct product $S=\prod_{n} F_{n}$. The direct sum $I=\oplus_{n} F_{n}$ is an ideal of the ring $S$, and so the ring $T=S / I$ is a commutative regular ring with an identity element. Let $L$ be the lattice $L(T)$ so that $L$ is complemented and modular.

To see that any two intervals in $L$ are lattice-isomorphic, it is enough (as in [9, Theorem 2.1]) to show that all intervals of the form $[0, A]$ are isomorphic, where $0 \neq A \in L$. So let $A$ be a nonzero principal ideal of $T$. Then $A$ is of the form $\left(S^{\prime}+I\right) / I$, where $S^{\prime}=\prod_{J} F_{n}$ and $J$ is an infinite subset of $N$. Thus $A \cong S^{\prime} /\left(S^{\prime} \cap I\right)$ and so $A$ and $T$ are isomorphic as rings. Since $A$ is a ring-direct-summand of $T$, it follows that the intervals $[0, A]$ and $[0, T]$ in $L$ are lattice-isomorphic. Hence any two intervals in $L$ are lattice-isomorphic.

Now suppose that $R$ is a regular bisimple ring such that $L(R) \cong L$. Since $T$ is a commutative (and so abelian) regular ring, [1, Theorem 3.4] shows that $L=L(T)$ is a distributive lattice. Conversely, since $L(R) \cong L$ is distributive, [1, Theorem 3.4] shows that the ring $R$ is abelian. Inasmuch as $R$ is also a simple ring (by [9, Lemma 1.2]), we see that $R$ must be a division ring (by [1, Theorem 3.2]). But then $L(R)=\{0, R\}$ which is a contradiction, since $L(R) \cong L$ and $|L|>2$.

The construction of the above example shows that regular bisimple rings $R$ are not characterized by the property that any two intervals in $L(R)$ are lattice-isomorphic. The following lemma pinpoints the property of regular bisimple rings which was missing in the ring $T$ in the example.

Lemma 1.2. Let $R$ be a regular ring. Then $R$ is bisimple if and only if any two nonzero principal right ideals of $R$ are isomorphic as right $R$-modules.

Proof. Munn showed that bisimple rings have this property in [9, Lemma 1.2]. For 
the converse, suppose that any two nonzero principal right ideals of $R$ are isomorphic. Let $a, b$ be nonzero elements of $R$. By hypothesis, there is an isomorphism $\theta: b R \rightarrow a R$. Then $c=\theta(b)$ satisfies $a R=c R$ and $r(c)=r(b)$. Since $R$ is regular, it follows that $R c=R b$, and so $R$ is bisimple.

This characterization shows that regular bisimple rings are closely related to the strongly prime rings used by Goodearl and Handelman in [2]. A ring $R$ with identity is said to be (right) strongly prime with bound 1 (or $\mathrm{SP}(1)$ for short) if for each nonzero $a \in R$ there is some $x \in R$ with $r(a x)=0$. Goodearl and Handelman show that $R$ is an SP(1) ring if and only if $R_{R} \precsim a R$ for each nonzero $a \in R$. Thus by Lemma 1.2, a regular bisimple ring is always an SP(1) ring. It is not known whether a regular $\operatorname{SP}(1)$ ring $R$ need be bisimple (see [1, Open Problem 51]), but [2, Theorem 2.1] shows that $R$ is bisimple in the special case where $R$ is also right self-injective.

Viewed in terms of Lemma 1.2, Example 1.1 shows that a lattice isomorphism between the intervals $[0, A]$ and $[0, B]$ (in $L(R)$ ) need not force an $R$-module isomorphism between the principal right ideals $A$ and $B$. This problem can be overcome if, instead, we insist that $A$ and $B$ be perspective in $L(R)$. However, we cannot expect all nonzero pairs in $L(R)$ to be perspective since, if $A \subset B$, then clearly $A$ and $B$ cannot have a common complement. Condition (b) in the following theorem shows that, if we just insist that all independent nonzero pairs $A$ and $B$ be perspective, then we capture all regular bisimple rings, but that rings of $2 \times 2$ matrices over division rings also get caught in the net. On the other hand, condition (c) gives a perspectivity condition that can be imposed on all nonzero pairs in $L(R)$, and no extra rings are caught this time.

Theorem 1.3. Let $R$ be a regular ring and $L(R)$ its lattice of principal right ideals. The following conditions are equivalent:

(a) $R$ is bisimple;

(b) (i) if $L(R)$ has a largest element, then this element is not of the form $A_{1} \oplus A_{2}$ where $A_{1}, A_{2}$ are distinct atoms in $L(R)$, and

(ii) any two nonzero independent elements of $L(R)$ are perspective in $L(R)$;

(c) for any nonzero $A, B \in L(R)$ there are decompositions $A=A_{1} \oplus A_{2}$ and $B=$ $B_{1} \oplus B_{2}$ such that, for $i=1,2$, the elements $A_{i}$ and $B_{i}$ are perspective in $L(R)$.

Proof. (a) $\Rightarrow$ (b). Assume that $R$ is bisimple. Suppose first that $L(R)$ has a largest element which has the form $A_{1} \oplus A_{2}$ where $A_{1}, A_{2}$ are distinct atoms in $L(R)$. Then $R=A_{1} \oplus A_{2}$ and so $R$ is Artinian. Since $R$ is bisimple, it follows from [9, Theorem 1.4] that $R$ must be a division ring. Thus $R$ is itself an atom in $L(R)$. This contradiction shows that (b)(i) holds.

Now let $A, B$ be nonzero principal right ideals of $R$ such that $A \cap B=0$. Since $R$ is bisimple, there are elements $a, b \in R$ such that $A=a R$ and $B=b R$ while $R a=R b$. We shall show that $C=(a+b) R$ is a common complement for $A$ and $B$ in $A+B$. Clearly $A+C=B+C=A+B$. To see that $A \cap C=0$ suppose $x, y \in R$ with $a x=(a+b) y$. Then 
$a(x-y)=b y \in A \cap B=0$ and so $b y=0$. As $R a=R b$, we get $a y=0$ too. Hence $A \cap C=0$. Similarly $B \cap C=0$, and so $A$ and $B$ are perspective in $L(R)$.

(b) $\Rightarrow$ (c). Assume that (b) holds. Let $A, B$ be nonzero principal right ideals of $R$, and consider first the case where $A \subset B$.

We begin by showing that $A$ cannot be an atom in $L(R)$. Suppose, on the contrary, that $A$ is an atom and let $C$ be its complement in $B$. By hypothesis, $A$ and $C$ are perspective (since $A \cap C=0$ ), and so $C$ is also an atom. Thus $B$ is the join of two distinct atoms, and so cannot be the largest element of $L(R)$. Since $L(R)$ is relatively complemented and modular, it follows that there is some nonzero $D \in L(R)$ with $B \cap D=0$. But now $D$ is perspective to both $A$ and $B$. Since $A$ is an atom and $B$ is the join of two distinct atoms, we have the desired contradiction.

Hence there is a decomposition $A=A_{1} \oplus A_{2}$ where $A_{1}$ and $A_{2}$ are nonzero elements of $L(R)$. Let $B_{1}$ be a complement of $A_{1}$ in $B$ and let $B_{2}=A_{1}$ so that $B=B_{1} \oplus B_{2}$. Since $A_{1} \cap B_{1}=0$ and $A_{2} \cap B_{2}=0$, both pairs of elements $A_{i}, B_{i}(i=1,2)$ are perspective in $L(R)$ as required.

A similar decomposition works if $B \subset A$, so suppose finally that $A \not \subset B$ and $B \not A$. Then there are decompositions

$$
\begin{aligned}
& A=(A \cap B) \oplus A_{1} \\
& B=(A \cap B) \oplus B_{1}
\end{aligned}
$$

where $A_{1}, B_{1}$ are nonzero elements of $L(R)$. Notice that $A \cap B \cap\left(A_{1}+B_{1}\right)=0$. Since $A_{1} \cap B_{1}=0$ our hypothesis implies that $A_{1}$ and $B_{1}$ are perspective in $L(R)$. We claim that $A$ and $B$ are also perspective in $L(R)$. Indeed, let $C$ be a common complement of $A_{1}$ and $B_{1}$ in $A_{1}+B_{1}$. Then clearly $A+C=B+C=A+B$. Also

$$
\begin{aligned}
A \cap C & =A \cap C \cap\left(A_{1}+B_{1}\right) \\
& =C \cap\left[A_{1}+(A \cap B)\right] \cap\left(A_{1}+B_{1}\right) \\
& =C \cap\left\{A_{1}+\left[(A \cap B) \cap\left(A_{1}+B_{1}\right)\right]\right\} \\
& =C \cap A_{1} \\
& =0
\end{aligned}
$$

and similarly $B \cap C=0$. Thus $A$ and $B$ are indeed perspective, and so in this case we can use the decompositions $A=A \oplus 0$ and $B=B \oplus 0$ to complete the proof that (c) holds.

(c) $\Rightarrow($ a). This follows from Lemma 1.2 since perspective modules are isomorphic.

Remark 1.4. We cannot omit condition (i) in (b) of the above theorem. Indeed if $R$ is the ring of $2 \times 2$ matrices over a division ring, then $L(R)$ satisfies (b)(ii), but $R$ is not a 
bisimple ring (because of Lemma 1.2). It is easy to see that this is the only extra class of regular rings which satisfy (b)(ii).

Remark 1.5. Let $R$ be a regular bisimple ring. Then the proof of $(b) \Rightarrow(c)$ in Theorem 1.3 shows that a sort of trichotomy law holds in $L(R)$ : for any $A, B \in L(R)$ we have either $A \subset B$ or $B \subset A$ or $A$ and $B$ are perspective in $L(R)$.

Conditions (b) and (c) are essentially lattice-theoretic conditions on the lattice $L(R)$, and indeed the proof that $(b) \Rightarrow$ (c) would carry over for any relatively complemented modular lattice $L$. Conversely, we could use [11, Theorem 3.6, p. 21] to show that (c) $\Rightarrow$ (b) for such an $L$. If $L$ has a greatest element, then the following result shows how von Neumann's co-ordinatization theorem can be used to find a regular bisimple ring $R$ such that $L(R) \cong L$. If you like, this gives a partial affirmative answer to the obvious modification of Munn's question [9, p. 185].

Corollary 1.6. Let $L$ be a complemented modular lattice such that

(i) the largest element of $L$ is not the join of two distinct atoms, and

(ii) any two nonzero independent elements of $L$ are perspective in $L$.

Then there is a regular bisimple ring $R$ (with identity) such that $L(R) \cong L$. Conversely, if $R$ is a regular bisimple ring with identity, then $L(R)$ is a complemented modular lattice satisfying (i) and (ii).

Proof. If $L$ has just two elements 0,1 then any division ring $R$ will give $L(R) \cong L$. So suppose $L$ has more than two elements. To use von Neumann's co-ordinatization theorem [11, Theorem 14.1, p. 208] we need to show that $L$ has order (at least) 4. That is, we need to find 4 independent, pairwise perspective elements $A_{1}, A_{2}, A_{3}, A_{4}$ in $L$ such that $A_{1} \oplus A_{2} \oplus A_{3} \oplus A_{4}=1$, the largest element of $L$.

Let $A \in L$ with $A \neq 0,1$ and let $A^{\prime}$ be a complement of $A$. As in the proof of Theorem $1.3(\mathrm{~b}) \Rightarrow(\mathrm{c})$, we see that $A \subset 1$ implies that $A$ is not an atom in $L$. Similarly $A^{\prime}$ cannot be an atom. Hence there are decompositions $A=A_{1} \oplus A_{2}$ and $A^{\prime}=A_{3} \oplus A_{4}$ where all the $A_{i}$ are nonzero. Since the $A_{i}$ are independent, condition (ii) ensures that $L$ has order 4 . By the co-ordinatization theorem there is a (unique) regular ring $R$ such that $L(R) \cong L$. By Theorem 1.3 this ring must be bisimple. The converse result is contained in Theorem 1.3 .

I do not know whether this result is also true for relatively complemented modular lattices.

\section{Products of idempotents}

Let $R$ be a regular bisimple ring. In this section we consider the multiplicative subsemigroup $S$ of $R$ generated by all the idempotents of $R$. We obtain a simple 
characterization of the elements of $S$, and calculate the depth of $S$. This continues a theme studied in [10], [3] and [4], where corresponding results were obtained in the cases where $R$ is a unit-regular ring, or where $R$ is regular and right self-injective. For regular bisimple rings $R$, we get an interesting dichotomy depending on whether or not $R$ has an identity element.

For the case where $R$ does not have an identity element, we need the following wellknown lemma.

Lemma 2.1. Let $R$ be a regular ring. For any $a \in R$ there is an idempotent $g \in R$ such that $a \in g R g$.

Proof. There is some $x \in R$ such that $a=a x a$. Then $e=a x$ is idempotent and $e a=a$. Again there is some $y \in R$ such that $a-a e=(a-a e) y(a-a e)$, and $f=(y-e y)(a-a e)$ is an idempotent. As $e$ and $f$ are orthogonal, $g=e+f$ is idempotent and it is easy to see that $a \in g R$.

Proposition 2.2. Let $R$ be a regular bisimple ring which does not have an identity element. Then every element of $R$ is a product of two idempotents, and so $R$ is a semiband of depth 2.

Proof. Let $a \in R$. By Lemma 2.1, there is an idempotent $g \in R$ such that $a \in g R g$. Since $g$ is not an identity element for $R$, there is a nonzero idempotent $h \in R$ such that $g h=h g=0$ (for example, if $z \in R$ with $z \neq g z$ then there is some $x \in R$ with $(z-g z) x(z-g z)=z-g z \neq 0$ and so $h=(z-g z)(x-x g)$ is such an idempotent $)$. By Lemma 1.2 we have $h R \cong g R$ and so (by [7, Proposition 4, p. 51]) there are elements $u \in g R h$ and $v \in h R g$ such that $g=u v$. Then

$$
a=[g+u][g+v(a-g)]
$$

is a product of two idempotents. (See [4, Example 2.15] for a matrix picture of this factorization.)

If every element of $R$ were idempotent, then $R$ would be commutative and so, by [9, Lemma 1.2(i)], would be a field. As this is impossible, $R$ must have depth exactly 2.

There is no direct parallel for this result in [10], [3] or [4] because the rings considered there all have identity elements. Munn gives some examples of regular bisimple rings without an identity in $[9,1.1]$.

On the other hand, if $R$ is a regular bisimple ring with an identity element, Proposition 2.3 below gives a chaiacterization and depth for the semigroup $S$ which is similar to those found in [3, Theorem 2.8] (or [10, Corollary 11]) and [4, Remark 1.7] for the case where $R$ is a regular right self-injective ring of type III. There is some overlap in these results: a simple, right self-injective ring of type III is always bisimple (this follows from Lemma 1.2 because of [2, Theorem 2.1] and [1, Corollary 10.17]). But there are many regular bisimple rings with identity which are not right self-injective. 
For example, most of the countable bisimple rings constructed by Munn in [9, Theorem 3.4] are not right self-injective, since a countable right self-injective ring is Artinian [8], and so a bisimple one must be a division ring by [9, Theorem 1.4].

Proposition 2.3. Let $R$ be a regular bisimple ring with identity and suppose that $R$ is not a division ring. Let $S$ be the subsemigroup of $R$ generated by all the idempotents of $R$. Then

$$
S=\{a \in R: a \text { is neither left nor right invertible }\} \cup\{1\}
$$

and $S$ has depth 3.

Proof. Clearly a product of proper $(\neq 1)$ idempotents cannot be left or right invertible. Suppose conversely that $a$ is a nonzero element of $R$ which is neither left nor right invertible. As $R$ is regular, we have $l(a) \neq 0$ and $r(a) \neq 0$. Also there are idempotents $e, f \in R$ such that $a R=e R$ and $R a=R f$. Thus $a \in e R f$ while $l(a)=R(1-e)$ and $r(a)=(1-f) R$. We shall use [3, Lemma 2.6] to show that $a$ is a product of three idempotents in $R$. Hence we just have to find some $g \in R$ such that $e R \cap g R=0$ and $f R \cap g R=0$, yet $f R \leqq g R$.

By Remark 1.5 we have either $e R \subset f R$, or $f R \subset e R$, or else $e R$ and $f R$ are perspective. Suppose firstly that $e R \subset f R$, and consider $g=1-f$. Since $r(a) \neq 0$, Lemma 1.2 shows that $f R \cong g R$. Also $e R \cap g R \subseteq f R \cap g R=0$ and so we are finished in this case. Similarly, if $f R \subset e R$ then $g=1-e$ will do the trick. Finally suppose that $e R$ and $f R$ are perspective, and let $g R$ be their common complement in $R$. Then $g R$ is nonzero and so Lemma 2.1 again gives an isomorphism $f R \cong g R$. Thus in all three cases [3, Lemma 2.6] shows that $a$ is a product of three idempotents. Hence $a \in S$ and $S$ has depth at most 3 .

Now since $R$ is not a division ring, there is a decomposition $R=A_{1} \oplus A_{2}$ with $A_{1}, A_{2}$ both nonzero principal right ideals. By Lemma 2.1 we have $A_{1} \cong A_{2} \cong R$, and so $R \cong R \oplus R$ as right $R$-modules. Hence [3, Example 2.2] shows that $R$ contains an element which is a product of three idempotents but no fewer. Thus $S$ has depth exactly 3.

Remark 2.4. In terms of the depth of the semigroup generated by all the idempotents of $R$, Propositions 2.2 and 2.3 both represent much "better" behaviour than is observed in arbitrary regular 0 -bisimple semigroups. For example, let $X$ be the finite set $\{1,2, \ldots, n\}$ and consider the semigroup $\mathscr{T}(X)$ of all transformations on $X$. Let

$$
J=\{\alpha \in \mathscr{T}(X): \text { rank } \alpha \leqq n-1\}
$$

and

$$
I=\{\alpha \in \mathscr{T}(X): \operatorname{rank} \alpha<n-1\}
$$

which are both ideals of $\mathscr{T}(X)$. Then the Rees quotient semigroup $R=J / I$ is regular 
and 0 -bisimple. By Howie's results in [6] it follows that $R$ is itself idempotent-generated and has depth $\left[\frac{3}{2}(n-1)\right]$.

\section{REFERENCES}

1. K. R. Goodearl, Von Neumann Regular Rings (Pitman, 1979).

2. K. R. Goodearl and D. Handelman, Simple self-injective rings, Comm. Algebra 3 (1975), 797-834.

3. J. Hannah and K. C. O'Meara, Products of idempotents in regular rings II, J. Algebra 123 (1989), 223-239.

4. J. Hannah and K. C. O'Meara, Depth of idempotent-generated subsemigroups of a regular ring, Proc. London Math. Soc. 59 (1989), 464-482.

5. J. M. HowIE, An Introduction to Semigroup Theory (Academic Press, 1976).

6. J. M. HowIE, Products of idempotents in finite full transformation semigroups, Proc. Roy. Soc. Edinburgh Sect. A 86 (1980), 243-254.

7. N. JACOBson, Structure of Rings (American Mathematical Society, 1964).

8. J. Lawrence, A countable self-injective ring is quasi-Frobenius, Proc. Amer. Math. Soc. 65 (1977), 217-220.

9. W. D. Munn, Bisimple rings, Quart. J. Math. Oxford 32 (1981), 181-191.

10. K. C. O'Meara, Products of idempotents in regular rings, Glasgow Math. J. 28 (1986), 143-152.

11. J. von Neumann, Continuous Geometry (Princeton University Press, 1960).

Mathematics Department

UNIVERSITY OF CANTERBURY

Christchurch

New Zealand 